\title{
Chapter 14
}

\section{Measuring Cell-Cell Tugging Forces Using Bowtie-Patterned mPADs (Microarray Post Detectors)}

\author{
Daniel M. Cohen, Mike T. Yang, and Christopher S. Chen
}

\begin{abstract}
Cells generate traction forces upon adhesion to the extracellular matrix as well as to neighboring cells. These forces are important for the growth and maintenance of adhesion structures such as focal adhesions and adherens junctions, and may play roles in tissue development. Here, we describe a method for measuring the tugging force transmitted across the cell-cell junction between two paired cells.
\end{abstract}

Key words Cell-cell tugging force, Cell-cell junctions, Micropatterning, mPADs, Traction force

\section{Introduction}

The ability of cell-matrix and cell-cell adhesions to support transmission of mechanical forces (either cell-generated or externally applied) has been demonstrated through a variety of methods including traction force microscopy [1-4], atomic or molecular force microscopy [5-7], optical or magnetic bead traps [8-11], and molecular tension reporters [12-15]. These methods have been especially useful in demonstrating that cells develop forces in response to newly formed adhesions, as well as in testing how adhesions respond/remodel upon loading with externally applied forces. However, measurements of mechanical forces that are transmitted across endogenous cell-cell junctions, in particular, have eluded these tools. Here, we present a general strategy for measuring cellcell tugging forces between pairs of cells. This approach has been successfully applied to measuring how cell-cell tugging forces between endothelial cells respond to vasoactive compounds, as well as to Rho and Rac GTPase signaling agonists/antagonists [16].

Our method employs culturing cells on specialized substrates, mPADs, comprised of elastomeric posts whose deformations quantitatively report cellular traction forces $[4,16]$. These mPAD arrays 
are available as a resource to the broader research community upon request from the Chen lab. To increase both the yield of cell-cell contact and promote more uniform cell-cell adhesions, the mPADs are functionalized with bowtie-shaped islands of fibronectin [17]. In these patterns, cell-cell contact is geometrically biased towards the neck of the bowtie, whereas the limited adhesion area per bowtie selects against the formation of multicellular ( $>2$ cell) clusters. In this configuration, the tugging force exerted by one cell is counterbalanced by the traction forces in the adjacent, contacting cell (net forces are in equilibrium, and therefore sum to zero). Thus the summation of traction forces under one cell yields a resultant force vector that is equal in magnitude and opposite in direction to the cell-cell tugging force exerted by its neighbor.

Using this method, we have previously demonstrated that endothelial cells generate cell-cell tugging forces on the order of $40 \mathrm{nN}$, with an apparent stress of approximately $\mathrm{lnN} / \mu \mathrm{m}^{2}$ at sites of cell-cell contact [16]. Moreover, this tugging force was required for the maintenance of the adherens junction, a finding that has now been corroborated by experiments correlating force, myosin, and cell-cell junction assembly in several cell types [18-22]. The mPAD system is amenable to studying cell-cell tugging forces in a variety of adherent cell types and can be easily adapted to either end-point analyses to measurement of dynamic force responses to extracellular stimuli $[16,23]$.

\section{Materials}

\subsection{Bowtie Stamping and mPAD Reagents}

1. Sylgard 184 polydimethylsiloxane (PDMS; Dow Corning, Morgan Hill, CA, USA).

2. Bowtie-patterned silicon wafers, as well as MPAD arrays can be generated by end-users with access to photolithography and microfabrication capabilities. Alternatively, mPADs are available upon request from the Chen Lab.

3. Prepare $5 \mathrm{mg} / \mathrm{ml}$ fibronectin (BD Biosciences, San Jose, CA, USA) by dissolving $5 \mathrm{mg}$ of fibronectin in $1 \mathrm{ml}$ of sterile water. Aliquot and freeze at $-20{ }^{\circ} \mathrm{C}$.

4. Prepare 2 \% F127 Pluronics (Sigma-Aldrich, St. Louis, MO, USA) stock solution by dissolving $2 \mathrm{~g}$ of F127 Pluronics in $100 \mathrm{ml}$ of distilled water in a bottle top $0.22 \mu \mathrm{m}$ filter. Allow Pluronics to dissolve and filter by gravity flow overnight. Dilute 1:10 into phosphate buffered saline (PBS) for a $0.2 \%$ working solution.

5. MatTek Petri dishes $(35 \mathrm{~mm}$, with $20 \mathrm{~mm}$ holes; MatTek Corporation, Ashland, MA, USA). 
6. Prepare DiI stock solution by dissolving $25 \mathrm{mg}$ of DiI (1,1'-dioleyl-3,3,3',3'-tetramethylindocarbocyanine methane sulfonate) (Life Technologies, Grand Island, NY, USA) in $500 \mathrm{ml}$ ethanol. Remove aggregates using a $0.22 \mu \mathrm{m}$ filter. Store at $4{ }^{\circ} \mathrm{C}$ in a dark or foil-wrapped bottle to protect from light.

\subsection{Immunostaining Reagents}

1. Add $10 \mathrm{ml}$ of $16 \%$ paraformaldehyde to $26 \mathrm{ml}$ of distilled water, and $4 \mathrm{ml}$ of $10 \times$ PBS to make a $4 \%$ solution. Add $100 \mathrm{ml}$ of $10 \%(\mathrm{v} / \mathrm{v})$ Triton X-100 to $10 \mathrm{ml}$ of $4 \%$ paraformaldehyde to make permeablilization/fixation solution.

2. Dissolve $5 \mathrm{ml}$ of goat serum (Life Technologies) in $45 \mathrm{ml}$ of PBS to make $10 \%(\mathrm{v} / \mathrm{v})$ blocking buffer.

3. Anti- $\beta$-catenin (BD Biosciences) is diluted $1: 100$ in $10 \%$ blocking buffer.

4. AlexaFluor 647 goat anti-mouse secondary antibody (Life Technologies) is provided as a $2 \mathrm{mg} / \mathrm{ml}$ solution. Use at $1: 400$ to $1: 800$ in $10 \%$ blocking buffer.

5. Dissolve AlexaFluor 488 phalloidin (Life Technologies) in $1.5 \mathrm{ml}$ of methanol. Dilute 1:100 in $10 \%$ blocking buffer. Dissolve $10 \mathrm{mg}$ of DAPI in $2 \mathrm{ml}$ of water to yield $5 \mathrm{mg} / \mathrm{ml}$ stock solution. Use at 1:2,000 dilution in $10 \%$ blocking buffer for immunostaining.

6. Fluoromount G (Electron Microscopy Sciences).

\section{Methods}

\subsection{Generation of mPAD Substrates}

For the purposes of simplicity, herein we provide a brief overview of the generation of silicon wafers that serve as templates for mPAD arrays. Design and fabrication of novel mPAD arrays requires users to have access to advanced microfabrication facilities and interested readers are referred elsewhere for detailed protocols [24-26].

Briefly, a photomask is generated containing the micropillar features in a regular square or hexagonal array. This photomask is used to pattern SU-8 photoresist that has been spin-coated on to a silicon wafer. Using a mask aligner and UV light source, the SU-8 becomes crosslinked by UV light in regions that are not protected by the photomask. Noncrosslinked SU-8 is removed, leaving behind micropillars of $7-12 \mu \mathrm{m}$ height and a $3-6 \mu \mathrm{m}$ diameter (specified by the features in the photomask). High-density mPAD arrays comprised of $2 \mu \mathrm{m}$ posts, with $4 \mu \mathrm{m}$ post-to-post spacing, have been generated using SU-8 photopatterning, but they require a more sophisticated process [24]. These silicon masters are used to cast negative PDMS molds (where the features are recessed wells). These negative PDMS molds are then used to cast 


\subsection{Functionalization of mPADs with Bowtie Micropatterns}

PDMS-based replicas of the micropillar substrates; herein referred to as mPAD substrates.

Silicon wafers for bowtie patterns use a similar SU-8 photopatterning approach, but do not require the generation of negative PDMS molds. Instead, the silicon wafers contain recessed wells in the shape of bowties, and PDMS stamps that contain the bowtie patterns are cast directly from the bowtie master (Subheading 3.2).

1. Prepare PDMS stamps as follows. Mix $60 \mathrm{~g}$ PDMS with $2 \mathrm{~g}$ curing agent in a plastic cup. Stir vigorously with a pipette for 3-5 min.

2. Degas PDMS solution in a vacuum desiccator until air bubbles are completely removed (30-60 $\mathrm{min}$ ).

3. Pour a 6-7 $\mathrm{mm}$ thickness layer of PDMS over a silicon wafer containing bowtie-shaped micropatterns in an aluminum dish (see Note 1). Make sure bubbles introduced while pouring the PDMS dissipate before proceeding to next step.

4. Bake stamps for $15 \mathrm{~min}$ at $110^{\circ} \mathrm{C}$ to cure the PDMS. Cool on bench for approximately $5 \mathrm{~min}$.

5. Gently peel PDMS stamps from silicon wafer and trim away excess PDMS. Cut stamps into squares sized-matched to the mPAD substrates (typically $21 \times 21 \mathrm{~mm})($ see Note 2$)$. Using a razor blade, notch the nonfeatured side of the stamp to indicate the bottom. Sterilize stamps by briefly $(2-5 \mathrm{~min})$ rinsing in $70 \%$ ethanol.

6. Dilute fibronectin to $50 \mu \mathrm{g} / \mathrm{ml}$ in sterile water (see Note 3). In a cell culture hood, place the stamps in a deep petri dish. Add the fibronectin solution in a dropwise fashion to the top (featured side) of the PDMS stamp. Gently drag droplets using a micropipettor until drops merge into a continuous surface covering the entire stamp. 50-100 $\mu \mathrm{l}$ of solution is needed to cover each stamp. Coat stamps for $60 \mathrm{~min}$ at room temperature.

7. Flood stamps with sterile water. Pick up stamps with tweezers and rinse twice more in a bath of sterile water. Dry stamps completely with compressed nitrogen (air gun). Place stamps face up in a fresh petri dish.

8. Render mPAD substrates hydrophilic by UV-ozone treatment in a UVO cleaner (model no. 342, Jelight, Irvine, CA, USA) for $7 \mathrm{~min}$.

9. Transfer mPADs from the UVO cleaner into a tissue culture hood. Position bowtie stamps onto mPADs using tweezers. Align stamp with one edge of the mPAD and allow stamp to slowly fall onto the substrate. Conformal contact can be verified by visual inspection of the substrates. From the perspective of an acute angle, a diffraction pattern should appear at the stamp:mPAD interface. If an incomplete pattern is present, 
apply gentle pressure on the stamp using tweezers until conformal contact is achieved. Allow $15 \mathrm{~s}$ for protein transfer.

10. Submerge mPADs in $1.4 \mathrm{ml}$ of $100 \%$ ethanol (mPADs should either be attached to a Mattek dish for live cell imaging or placed in a $35 \mathrm{~mm}$ petri dish). Displace stamp from mPADs with a gently knocking motion (do not peel the stamp off as this can collapse the mPADs). Set stamps aside ( see Note 4).

11. Add $0.6 \mathrm{ml}$ of water to the mPAD dish and swirl to mix. Remove this $70 \%$ ethanol solution by aspiration and rinse $3 \times$ with sterile milliQ water. Do not allow the substrates to dewet (keep samples flat, not tilted, during aspiration steps).

12. Label the PDMS with DiI. Dilute a $50 \mu \mathrm{g} / \mathrm{ml}$ DiI stock solution to $5 \mu \mathrm{g} / \mathrm{ml}$ working concentration using sterile milliQ water $(2 \mathrm{ml}$ per substrate). Add to $\mathrm{MPADs}$ and incubate for $60 \mathrm{~min}$. Cover sample with aluminum foil to protect from light in all subsequent steps.

13. Aspirate DiI solution and rinse $3 \times$ with milliQ water. Add $0.2 \%$ Pluronics F-127 to passivate the nonfunctionalized regions of PDMS. Allow 30-60 min for adsorption of Pluronics.

14. Rinse $3 \times$ with milliQ water. mPADs can now be used for cell seeding or stored in PBS at $4{ }^{\circ} \mathrm{C}$ for up to 1 week until cells are ready.

\subsection{Seeding MPADs} with Cells
1. Aspirate PBS or water from mPADs and replace with $2 \mathrm{ml}$ of cell culture medium.

2. Trypsinize and resuspend cells to $20,000-100,000$ cells $/ \mathrm{ml}$. Add $0.5 \mathrm{ml}$ of cell suspension to the mPADs (to achieve a seeding density of $1,000-5,000$ cells $/ \mathrm{cm}^{2}$ ). Be sure to disrupt or remove any clumps of cells to avoid occupancy of bowties by more than two cells.

3. Allow cells to adhere to $\mathrm{mPAD}$ substrates for $10 \mathrm{~min}$ in a tissue culture incubator (see Note 5).

4. Rinse away excess nonadherent cells. If mPADs are attached to a Mattek dish, aspirate and replenish medium $3 \times$ or until no floating cells are observed. Alternatively, mPADs on glass coverslips can be successively transferred to new $35 \mathrm{~mm}$ dishes with fresh culture medium to remove nonadherent cells.

5. Return mPADs to tissue culture incubator and allow the cells to spread overnight.

6. If desired, change medium per experimental needs. Serum starvation $(0.1-0.5 \%$ serum $)$ is recommended to observe changes in traction and tugging forces in response to the addition of soluble agonists. Serum starvation periods of 18-24 h are usually adequate to reduce cellular contractility to basal levels. 


\subsection{Immunostaining}

7. Upon stimulation, changes in cell traction and tugging forces occur within 2-30 min. Treat cells with the manipulation or stimulus of interest (or a vehicle control), rinse briefly with PBS, and fix at the desired end-point.

8. Fix/permeabilize cells with $2 \mathrm{ml}$ of room temperature $4 \%$ paraformaldehyde containing $0.1 \%$ Triton X-100 for $3 \mathrm{~min}$, followed by an additional fixation in $4 \%$ paraformaldehyde for $15 \mathrm{~min}$ ( see Note 6). If multiple conditions are being tested in parallel, be sure to stagger the fixation times. Force measurements are quite sensitive to variations in rinsing and fixation times. Alternatively, time lapse imaging of mPAD displacements can be performed during this time (see Note 7).

1. Aspirate paraformaldehyde, and rinse for $5 \mathrm{~min}$ with PBS. Prepare samples for immunofluorescence by blocking with $10 \%$ goat serum (in PBS) or an equivalent blocking agent.

2 . Immunostain for cell-cell junctions using an appropriate antibody. We recommend mouse anti- $\beta$-catenin at a 1:100 dilution in blocking buffer (10\% goat serum). For antibody incubations, invert mPADs onto a $200 \mu \mathrm{l}$ droplet of diluted primary antibody on a piece of parafilm. Be sure to avoid contacting mPADs directly with tweezers (handle edge of coverslip only) to avoid collapse of the posts. Incubate mPADs with primary antibody for $\mathrm{l} \mathrm{h}$ at room temperature.

3. Place mPAD substrates in a $35 \mathrm{~mm}$ dish and rinse $4 \times$ for $5 \mathrm{~min}$ with PBS.

4. Immunostain mPADs with secondary antibody for $\mathrm{l} h$ at room temperature as in step 2. Cover samples to protect from light.

5. Place mPAD substrates in a $35 \mathrm{~mm}$ dish and rinse $4 \times$ for $5 \mathrm{~min}$ with PBS.

6. Optional: Stain cells with fluorescent phalloidin to visualize the actin cytoskeleton and determine cell boundaries during mPAD quantification. Counterstain nuclei with DAPI. Stain for $20 \mathrm{~min}$ in a $200 \mu \mathrm{l}$ droplet as in step 4 (if desired, this step can be combined with step 4 , as extending the incubation to $>20 \mathrm{~min}$ is not problematic). Cover samples to protect from light.

7. Place $\mathrm{mPAD}$ substrates in a $35 \mathrm{~mm}$ dish and rinse $4 \times$ for $5 \mathrm{~min}$ with PBS.

8. Mount mPADs on slide using Fluoromount G. Use a small drop to adhere mPAD face-up to glass slide. Cover top of mPADs with a few drops of Fluoromount $G$ and seal with a fresh $22 \mathrm{~mm}$ glass coverslip. Allow Fluoromount to cure for $4 \mathrm{~h}$. Fluorescent microscopy imaging should be done with an inverted microscope. 


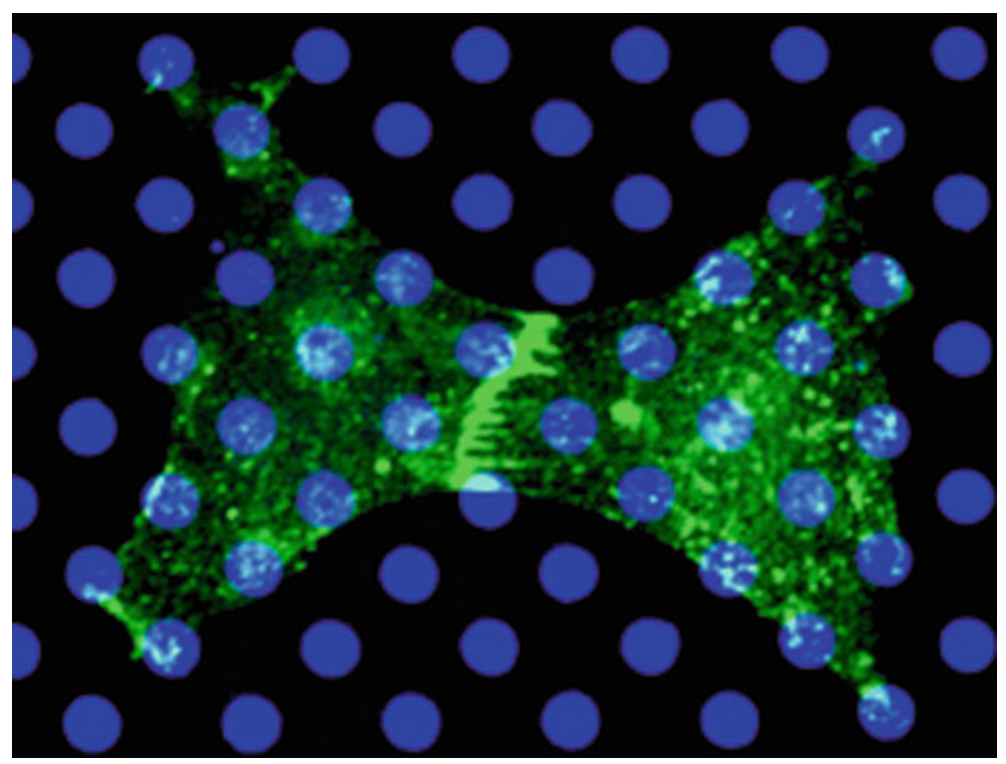

Fig. 1 Cell-cell junctional staining of bowtie-patterned endothelial cells on mPADs. Dil-stained microposts are shown in blue and $\beta$-catenin immunostaining shown in green. Note cell-cell junction in center bowtie. Diffuse cytoplasmic $\beta$-catenin in cell body is removed by thresholding in subsequent image processing steps

\subsection{Image \\ Acquisition and Quantification of Cell-Cell Junctions}

1. Using an inverted microscope, collect multi-channel images to define the position of the cell nucleus (DAPI stain), the cell boundaries (phalloidin, green channel), and the cell-cell junctions (anti- $\beta$-catenin, far red/infrared channel). Oil immersion objectives are recommended with a magnification of at 40-63×. Microscopy can be done either in epifluorescence or confocal modes (see Notes 8 and 9). An example of typical staining pattern of $\beta$-catenin overlaid on the micropost channel is provided in Fig. 1.

2. Acquire images of microposts tips using the DiI signal (red channel); henceforth the "top image". Make sure that the image includes two peripheral rows of posts surrounding the bowtie region ( $\mathrm{see}$ Note 10). It is best to collect at least 20-30 sets of images of bowties in steps 1 and 2 .

3. Quantify cell-cell junction size and brightness using appropriate image analysis software (e.g. Matlab, MetaMorph). For epifluorescence images, perform a flatfield correction to correct for inhomogeneities in illumination, followed by pixels thresholding to select the brightest $25 \%$ of pixels in the $\beta$-catenin channel. The resultant image is then binarized and all pixels are summed to generate an area measurement for cell-cell junctions. In addition, mean pixel intensity can be determined by averaging the intensity values of the $\beta$-catenin pixels in the thresholded image. 
3.6 Force Detection Algorithm
A MATLAB-based program for traction force measurements is available upon request from the Chen Lab. Here we simply describe the general approach and the extra steps required for determination of cell tugging force.

1. Although the mPADs conform to a regular array (either square or hexagonally packed), this array may be rotated arbitrarily in any given image due to variability in how the sample was mounted relative to the camera's field of view on the microscope. Images should be rotated to match a single Cartesian coordinate system, such that the rows of microposts align with the horizontal $X$-axis and vertical $\Upsilon$-axis.

2. Generate an ideal grid to predict the expected location for all posts using linear interpolation. In each row of microposts, "guide posts" are selected from the left and right side of the bowtie. Using these guide posts, an equation is determined for the best-fit line through the center of the posts. Since the spacing between posts is fixed, the number of posts, and position of these posts between the guide posts can be determined by the post-to-post spacing constant (see Note 11).

3. Determine which posts are in contact with a cell. Overlay the phalloidin and/or $\beta$-catenin images on the mPAD (DiI) image. If desired, a registration step can be done to correct for translational shifts between the images acquired in different fluorescence channels. All posts positioned under the cell body or associated with cellular protrusions such as filopodia are considered to be in contact with a cell.

4. Find the centroid of the posts and determine deflections. Post positions are determined by thresholding the DiI fluorescence image. An appropriate threshold value will yield pixel segments that approximate the cross-sectional area of the posts. At this point, the centroid can be determined for segmented group of pixels (i.e. each post). Subtract the experimentally determined centroid from the undeflected centroid location as determined by the ideal grid (Subheading 3.5, step 2) to calculate displacements per post. An illustration of the traction force vectors mapped on to the $\mathrm{mPAD}$ micrographs is provided in Fig. 2a.

5. Vectorial displacements can be converted to traction force vectors using the spring constant for the posts (see Note 12). From the traction force vectors, the magnitude and direction of forces are known. To calculate tugging force, all of the traction force vectors under a given cell are summed (Fig. 2b). In the case of an isolated cell, the $X$ and $\Upsilon$ components of force should sum to zero (equilibrium state). However, in the bowtie, the sum of the traction forces under each cell should be summed with the tugging force to achieve the equilibrium 


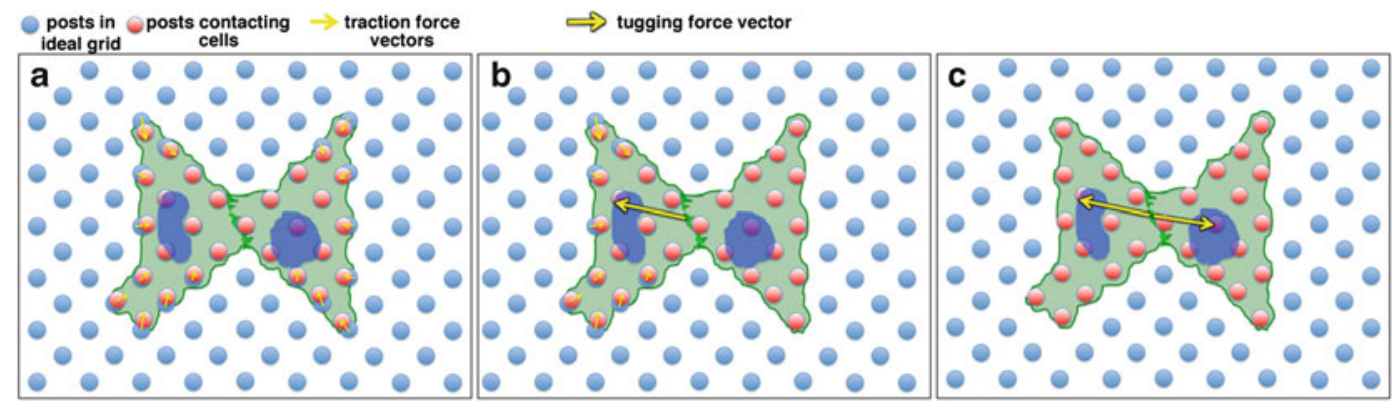

Fig. 2 Determination of cell-cell tugging force in bowtie-patterned cells on mPADs. (a) Top-down view of cells on mPADs. Each micropost is represented by a circle, showing either its position in the ideal grid (blue) versus its experimentally measured position (red). For simplicity, experimentally measured positions are limited here to those posts that are in contact with bowtie-patterned cells. The distance between centroids of the blue versus red posts is used to calculate traction force vectors (yellow arrows) using the spring constant of the posts (see Note 12). (b) Determination of tugging force vector for the cell located in the left half of the bowtie. Considering only the traction forces in the left cell, the $x$ and components of the vectors are summed to yield a resultant traction force vector. The tugging force vector is then plotted as the equal, and opposite, force vector such that the net forces sum to zero. (c) The process in panel $\mathbf{b}$ is repeated for the cell located in the right half of the bowtie, yielding an independent tugging force vector for each cell. Tugging force per bowtie is reported as the average magnitude of the two tugging force vectors

state. Therefore the tugging force is calculated as an equal and opposite force to the resultant vector for all traction forces in the neighboring cell ( see Note 13). This process is repeated for each cell in the bowtie pair to generate two independent tugging force vectors (Fig. 2c). Average tugging force per bowtie is reported as the average magnitude of the two tugging force vectors.

\section{Notes}

1. We strongly suggest the use of bowtie patterns to enhance the yield of paired cells with well-defined cell-cell contacts (other geometric patterns, or featureless, flat stamps can be used in principle). Some optimization may be needed to select an appropriate bowtie pattern for a given cell type; ideally, cell spreading in each half of the bowtie should approximate the average area occupied by a cell when grown to confluency (typically $40-50 \%$ of the area of maximal cell spreading observed in subconfluent conditions). Bowtie geometries are comprised of two isosceles triangles (2:2:3 dimensional ratio) that join at their vertices. Upon transfer of the bowtie patterns to the MPAD surfaces, the gap of nonadhesive surface that cells need to span in order to form cell-cell contacts will depending on micropost spacing (typically on the order of 4-9 $\mu \mathrm{m}$ ). 
2. For a detailed demonstration of generating and using PDMS stamps, readers are referred to ref. 27.

3. Fibronectin solutions need not be made freshly every time; however, dilute fibronectin solutions denature over time and should not be kept more than $2-3$ weeks at $4{ }^{\circ} \mathrm{C}$. Other matrix proteins such as collagen are compatible with microcontact printing, and can be used in lieu of fibronectin.

4. PDMS stamps can be reused in future experiments. After each use, sonicate for $5 \mathrm{~min}$ in $95 \%$ ethanol to remove any residual fibronectin from the stamps.

5. Timing for cell adhesion to mPAD substrates can be dependent on several factors (e.g. cell type, medium composition, type of extracellular matrix in bowties, quality of stamping). If cells are not attached by $10 \mathrm{~min}$, monitor the attachment in 5 min intervals until at least half the patterns show cell attachment. If excessive pooling of cells occurs, gently rock samples to disperse cells evenly.

6. Simultaneous permeabilization and fixation is recommended to remove the diffuse cytoplasmic pool of $\beta$-catenin that can otherwise obscure imaging of cell-cell junctions. This permeabilization step does not appear to interfere with the contractility state of the cell.

7. Live cell microscopy, when available, offers several distinct advantages to end-point based analyses for measurement of tugging forces; it can avoid artifacts associated with changes in contractility during the fixation process and enable insight into dynamics of tugging forces. However, tugging force analysis on live cells requires a vital label for cell-cell junctions. For example, in endothelial cells, adenoviral delivery of GFP-VEcadherin was used to visualize the junctions [28]. Choice of label and mode of delivery need to be optimized for the cell type of interest.

8. If available, confocal microscopy is especially useful for imaging the cell-cell junctions by eliminating out-of-plane fluorescence. However, we find that it is possible to compensate for shortcomings of epifluorescence using image processing described here. Access to confocal imaging obviates the need for these processing steps.

9. When collecting images, adjust the z-position to select the appropriate focus position for each fluorescent marker (be sure to capture a phalloidin image near the base of the cell to determine which microposts are in contact with a cell). Be sure to select bowties that have exactly two cells and show a distinct 
cell-cell junction at or near the neck of the bowtie. It is critical to use a constant exposure time for the $\beta$-catenin and DiI channels or subsequent quantitative analyses will not be possible.

10. The force detection algorithm requires information about nondeflected posts. Therefore, it is critical to select a magnification in which this top image contains at least two rows of nondeflected posts surrounding the bowtie-patterned cells. If necessary, switch to a lower magnification such that this requirement is met. The optimal focal plane for top images can be found by focusing down from the plane of the cell until the peripheral microposts (non-cell contacting) come sharply into view (post-tips should be perfectly circular, with the notable exception of severely deflected posts under a cell).

11. This calculation requires prior knowledge of the scale size for each image (i.e. how many microns per pixel). In principle, linear interpolation can be applying to incorporate positional information on both the horizontal and vertical axes; however, this approach does not significantly increase accuracy of the ideal grid and may even be less robust in cases where uneven illumination or other image distortions introduce a systematic error in micropillar position across the field of view.

12. Forces can be predicted by Hooke's law; $F=k \times X$, where $k$ represents the spring constant and $X$ is the measured displacement. The spring constant of the post is determined by the equation, $k=3 \times E \times I / L^{3}$, where $E$ is the elastic modulus, $I$ is the area moment of inertia, and $L$ is the length of the post. Spring constants of mPADs have also been empirically determined using micromanipulators to apply known forces to the posts.

13. The inference that the tugging force acts as a balancing force for the summed traction forces is only valid for pairs of cells. If more than two cells are in contact, it is impossible to know how the balancing forces imparted by the cell-cell tugging forces are distributed between them (there is no unique solution to the force balance equation). Therefore, this method cannot be generalized to multicellular clusters.

\section{Acknowledgements}

This work was supported by NIH grants HL73305 and through the RESBIO program (Integrated Technology Resource for Polymeric Biomaterials, NIH grant EB001046). 


\section{References}

1. Lee J, Leonard M, Oliver T (1994) Traction forces generated by locomoting keratocytes. J Cell Biol 127:1957-1964

2. Pelham RJ, Wang Y (1999) High resolution detection of mechanical forces exerted by locomoting fibroblasts on the substrate. Mol Biol Cell 10:935-945

3. Balaban NQ, Schwarz US, Riveline D et al (2001) Force and focal adhesion assembly: a close relationship studied using elastic micropatterned substrates. Nat Cell Biol 3:466-472

4. Tan JL, Tien J, Pirone DM et al (2003) Cells lying on a bed of microneedles: an approach to isolate mechanical force. Proc Natl Acad Sci USA 100:1484-1489

5. Perret E, Leung A, Feracci $\mathrm{H}$ et al (2004) Trans-bonded pairs of E-cadherin exhibit a remarkable hierarchy of mechanical strengths. Proc Natl Acad Sci USA 101:16472-16477

6. Baumgartner W, Hinterdorfer P, Ness W et al (2009) Cadherin interaction probed by atomic force microscopy. Proc Natl Acad Sci USA 97:4005-4010

7. Panorchan P, Thompson MS, Davis KJ et al (2006) Single-molecule analysis of cadherinmediated cell-cell adhesion. J Cell Sci 119:66-74

8. Potard US, Butler JP, Wang N (1997) Cytoskeletal mechanics in confluent epithelial cells probed through integrins and E-cadherins. Am J Physiol 272:C1654-1663

9. Ko KS, Arora PD, McCulloch CA (2001) Cadherins mediate intercellular mechanical signaling in fibroblasts by activation of stretchsensitive calcium-permeable channels. J Biol Chem 276:35967-35977

10. Felsenfeld DP, Choquet D, Sheetz MP (1996) Ligand binding regulates the directed movement of betal integrins on fibroblasts. Nature 383:438-440

11. Galbraith CG, Yamada KM, Sheetz MP (2002) The relationship between force and focal complex development. J Cell Biol 159:695-705

12. Ohashi T, Kiehart DP, Erickson HP (2002) Dual labeling of the fibronectin matrix and actin cytoskeleton with green fluorescent protein variants. J Cell Sci 115:1221-1229

13. Smith ML, Gourdon D, Little WC et al (2007) Force-induced unfolding of fibronectin in the extracellular matrix of living cells. PLoS Biol $5: \mathrm{e} 268$

14. Kong HJ, Polte TR, Alsberg E et al (2005) FRET measurements of cell-traction forces and nano-scale clustering of adhesion ligands varied by substrate stiffness. Proc Natl Acad Sci USA 102:4300-4305
15. Grashoff C, Hoffman BD, Brenner MD et al (2010) Measuring mechanical tension across vinculin reveals regulation of focal adhesion dynamics. Nature 466:263-266

16. Liu Z, Tan JL, Cohen DM et al (2010) Mechanical tugging force regulates the size of cell-cell junctions. Proc Natl Acad Sci USA 107:9944-9949

17. Nelson CM, Liu WF, Chen CS (2007) Manipulation of cell-cell adhesion using bowtie-shaped microwells. Methods Mol Biol 370:1-10

18. Maruthamuthu V, Sabass B, Schwarz US et al (2011) Cell-ECM traction force modulates endogenous tension at cell-cell contacts. Proc Natl Acad Sci USA 108:4708-4713

19. Miyake $\mathrm{Y}$, Inoue $\mathrm{N}$, Nishimura $\mathrm{K}$ et al (2006) Actomyosin tension is required for correct recruitment of adherens junction components and zonula occludens formation. Exp Cell Res 312:1637-1650

20. Shewan AM, Maddugoda M, Kraemer A et al (2005) Myosin 2 is a key Rho kinase target necessary for the local concentration of E-cadherin at cell-cell contacts. Mol Biol Cell $16: 4531-4542$

21. Huveneers S, Oldenburg J, Spanjaard E et al (2012) Vinculin associates with endothelial VE-cadherin junctions to control forcedependent remodeling. J Cell Biol 196:641-652

22. Yonemura S, Wada $Y$, Watanabe $T$ et al (2010) alpha-Catenin as a tension transducer that induces adherens junction development. Nat Cell Biol 12:533-542

23. Yang MT, Reich DH, Chen CS (2011) Measurement and analysis of traction force dynamics in response to vasoactive agonists. Integr Biol (Camb) 3:663-674

24. Yang MT, Fu J, Wang YK et al (2011) Assaying stem cell mechanobiology on microfabricated elastomeric substrates with geometrically modulated rigidity. Nat Protoc 6:187-213

25. Sniadecki NJ, Chen CS (2007) Microfabricated silicone elastomeric post arrays for measuring traction forces of adherent cells. Methods Cell Biol 83:313-328

26. Desai RA, Yang MT, Sniadecki NJ et al (2007) Microfabricated post-array-detectors (mPADs): an approach to isolate mechanical forces. J Vis Exp 8:e311

27. Shen K, Qi J, Kam LC (2008) Microcontact printing of proteins for cell biology. J Vis Exp 22:e1065

28. Shaw SK, Bamba PS, Perkins BN et al (2001) Real-time imaging of vascular endothelialcadherin during leukocyte transmigration across endothelium. J Immunol 167:2323-2330 